\title{
Computer aided drug designing for proteasome inhibitor to find out potential drug candidate for multiple myloma
}

\begin{abstract}
Block the action of proteosome via Proteasome inhibitors. This inhibitors act as drug candidate for blocking the malfunctioning of proteosome. In market very few drugs available for the multiple myloma treatment in this finding we update very famous drug bortezomib for multiple myloma treatment via computational platforms and we found this drug bind via protein ligand interaction with favorable statical \& structural (Tables Below) computation platforms.
\end{abstract}

Keywords: Proteasome inhibitors, bortezomib, multiple myloma, ligand
Volume 4 Issue 5 - 2018

\author{
Vinita Srivastava,' Shashi Prabha Agrawal, ${ }^{2}$ \\ Swinder Jeet Singh Kalra, ${ }^{3}$ Alka Dubey ${ }^{4}$ \\ 1,3 Associate Professor from Department of Chemistry, D.A-V, \\ College Kanpur INDIA \\ ${ }^{2}$ Assistant Professor from Department of Zoology, D.A-V \\ College Kanpur, INDIA \\ ${ }^{4}$ Visiting Research Associate, in Bioinformatics Infrastructure \\ Facility (Funded by Dept. of Biotechnology, Govt. of India) of \\ Forest Research Institute, Dehradun, INDIA
}

Correspondence: Alka Dubey, Visiting Research Associate, in Bioinformatics Infrastructure Facility (Funded by Dept. of Biotechnology, Govt. of India) of Forest Research Institute, Dehradun, India, Email alkabioinfo964@gmail.com

Received:September 12, 2018| Published: October 23, 2018

\section{Introduction}

The incorporation of the first proteasome inhibitor, bortezomib, into anti-myeloma armamentarium can be considered a major milestone in treatment of multiple myeloma, greatly improving the response rates and overall survival in front-line and relapsed/ refractory settings. Although present in all cells, proteasomes are relatively abundant in multiple myeloma cells making that disease a target for proteasome inhibitors. The proteasome Protein activity regulation by synthesis and degradation maintains cellular metabolic integrity and proliferation. The proteasome, a multimeric protease complex, is central to cellular protein regulation by degrading many proteins, thus activating some pathways and shutting down others. Proteins conjugated to multiple units of the polypeptide ubiquitin are degraded by the proteasome. Widely studied inhibitors included: lacta-cystin, a streptomyces metabolite, which is metabolized to lactacystin b-lactone, the active proteasome inhibitor peptide aldehydes, such as carbobenzoxyl-leucinyl-leucinyl-leuc-inal- $\mathrm{H}$ (MG-132); boronic acid peptides and others Dipeptide boronic acid derivatives, potent proteasome inhibitors, could be administered to mice, and some were orally bioavailable and relatively stable under physiological conditions. Boronic acid peptides were known to inhibit serine proteases, whereas the dipeptide boronates had a high degree of selectivity for the proteasome and were not potent inhibitors of many common proteases. Critical to the clinical development of proteasome inhibitors was establishing a pharmacodynamic assay which measured the potency and duration of inhibition of the proteasomes in normal blood cells. In animal-model studies, the dipeptide boronic acid, PS-341, rapidly disappeared from the vascular compartment. An accurate pharmacodynamic assay was developed to supplement pharmacokinetic measurements. Both the chymo-tryptic and tryptic activities of the proteasome could be detected using fluorogenic kinetic assays. These fluorogenic kinetic assays were optimized for both whole blood and blood cells.

Treatment of myeloma has developed rapidly over the past two decades with the advent of proteasome inhibitors and immunomodulatory agents. Despite their dramatic impact on overall patient outcomes, there remains a high-risk group of patients, constituting $20-30 \%$ of all cases, who have not benefited to the same extent and continue to have poor outcomes. There is no single pathogenic mechanism that can currently be used to unify and define high-risk disease; instead, the common features are clinically aggressive behaviours, including therapy resistance, proliferation and evasion of apoptosis, which are the consequences of following several evolutionary trajectories driven by distinct gene-gene interactions. The key molecular hallmarks of high-risk disease biology include increased proliferation and the development of a high-risk ecosystem that facilitates both cancer cell survival and failure of the immune response. Genetic lesions underlie the key hallmarks of high-risk disease states and may occur as tumour initiating or progression events. No one of these lesions explains all high-risk disease, and in order to effectively target high-risk disease, we need to understand how these drivers arise, interact with each other and mediate their downstream effects. Pathologically, the high-risk biological states of multiple myeloma are the end stage of a multi-step clinical progression system typical of multiple myeloma comprising benign monoclonal gammopathy; an intermediate stage lacking clinical damage; multiple myeloma itself, which has a range of clinical behaviours; and an easily recognizable leukaemic phase wherein disease is no longer confined to the bone marrow. This progression system is driven by subclonal competition and selective pressures within the bone marrow microenvironment. ${ }^{2}$ The proteasome is a multisubunit protease complex with an apparent sedimentation coefficient of 20S. Two types of regulatory complexes, named PA700 and PA28, bind to both ends of the cylindrical 20S proteasome to form the dumbbell-like and football-like proteasomes, respectively. The former complex, named the $26 \mathrm{~S}$ proteasome, is a eukaryotic ATP-dependent protease and appears to be well organized as a large complex of 2 $\mathrm{MDa}$, consisting of approximately 40 polypeptides, to facilitate rapid proteolysis. It is assumed to be a protein "death machine", destroying a variety of cellular proteins that have acquired a specific degradation signal(s) such as a multiubiquitin chain. 
Bortezomib is an antitumor drug that competitively inhibits proteasome beta-1 and beta-5 subunits. While the impact of bortezomib on protein stability is known, the effect of this drug on intracellular peptides has not been previously explored. A quantitative peptidomics technique was used to examine the effect of treating human embryonic kidney 293 T (HEK293T) cells with 5-500nM bortezomib for various lengths of time (30minutes to 16 hours), and human neuroblastoma SH-SY5Y cells with $500 \mathrm{nM}$ bortezomib for 1 hour. Although bortezomib treatment decreased the levels of some intracellular peptides, the majority of peptides were increased by $50-500 \mathrm{nM}$ bortezomib. ${ }^{4}$

\section{Material \& methods}

Database NCBI (National Center for Biotechnology Information) ${ }^{5}$ PDB (Protein Data Bank). ${ }^{6}$ Drug Bank. ${ }^{7}$ Tools: BLAST (Basic Local Alignment Search Tool). ${ }^{8}$ Model validation: SAVES (Structural Analysis and Verification Server $)^{9}$ Model visualization: Chimera, Rasmol, Pymol, discovery studio, Binding site analysis: Qsite Finder, Pocket Finder, ${ }^{10}$ Dockingtool: Auto Dock, hex, PATCHDOCK, (hexserver.loria.fr/)Automated Docking Server: Online different type of docking server, The first step in methodology is collection of sequences data from NCBI. Sequence alignment: The protein sequences of multiple myloma (>sp|Q96EZ4.2|MYEOV_HUMAN Rec Name: Full=Myeloma-over expressed gene protein; Alt Name: Full=Oncogene in multiple myeloma) were obtained from NCBI/PDB after that the homology modeling of sequence is done then selection of the best model is done with the help of core region and model validation a binding site is also predicted via online tools then go for docking for identification of potential ligand with minimum energy. ${ }^{11}$

\section{Results and discussion}

Theresults analysis base on Sequence of Rec Name: Full=Myeloma- overexpressed gene protein; Alt Name: Full=Oncogene in multiple myeloma

$>$ sp|Q96EZ4.2|MYEOV_HUMAN Rec Name: Full=Myelomaoverexpressed gene protein; Alt Name: Full=Oncogene in multiple myeloma. $^{12}$

MALRICVTYTPALPIGLCTRCCLCLEQSPSWCHCLRGVSFLTF HLHQSVPLGDRDSLLMFTRQAGHFVEGSKAGRSRGRLCLSQ ALRVAVRGAFVSLWFAAGAGDRERNKGDKGAQTGAGLSQE AEDVD VSRARRVTDAPQGTLCGTGNRNSGSQSARVVGVAH LGEAFRV GVEQAISSCPEEVHGRHGLSMEIMWARMDVALRS PGRGLLAGAGALCMTLAESSCPDYERGRRACLTLHRHPTPH CSTWGLPLR VAGSWLTVVTVEALGGWRMGVRRTGQVGPT MHPPPVSGASPLLLHHLLLLLLIIILTC

Expectation Value $=0.001$, Search Tool $=$ blast, Mask Low Complexity=yes) via BLASTP .this blast mainly use for protein the results of Computer Aided Drug Designing of Proteosome Inhibitors to find the potential drug candidate for Multiple myloma based on different type potential parameters Homology modeling, also known as comparative modeling of protein, refers to constructing anatomic-resolution model of the "target" protein formats amino acid sequence and an experimental three-dimensional structure of a related homologous protein. In this project homology modeling completed with Geno3D \& Phyre it is an automatic web server for protein molecular

\section{Model validation}

Model validation completed with the help of SAVES server. It is type of online web server for model validation and for analyzing protein structure for validity and assessing how correct they are it's based on six programs Results: the Model 1 is pass by the SAVES server (Figure 1).
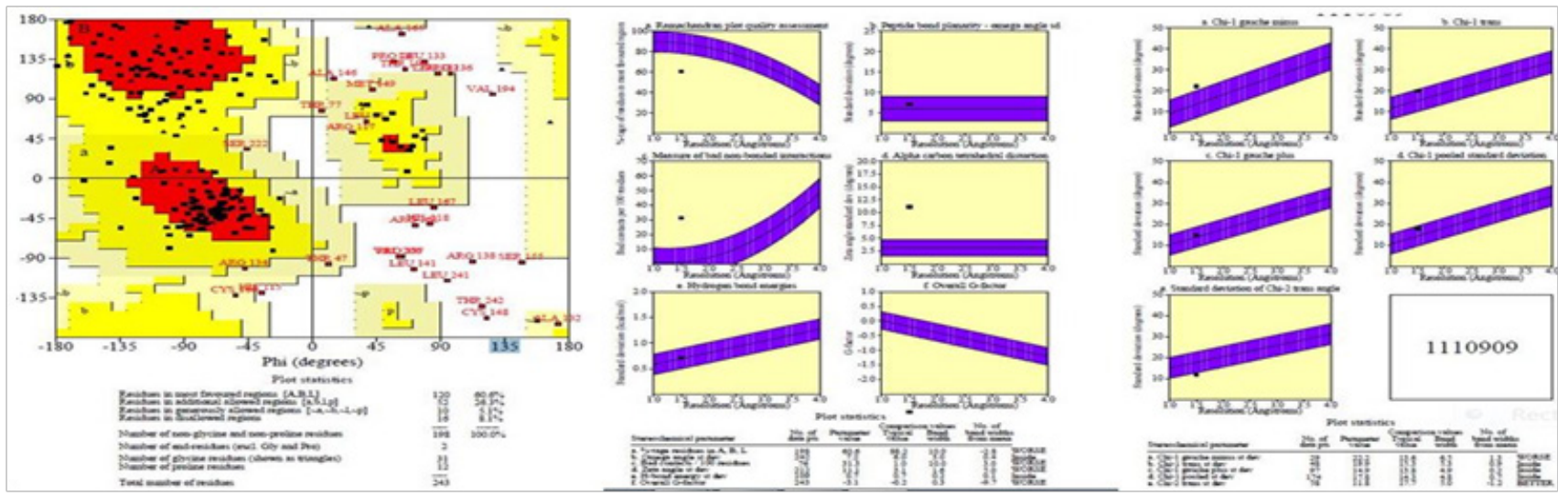

Figure I Ramchandran plot \& Free residues structures.

\section{Visualization}

Selected model analysis based on 2D and 3D structure visualization and the model visualization completed with the help of different type of software's for example: Chimera, Rasmol, and discovery studio computational predicted models via bioinformatics approaches and the method of homology modelling is based on the observations because that's protein tertiary structure is better conserved than amino acid sequence (Figures 2-4). ${ }^{13}$

\section{Binding site prediction}

According Steps selected protein model submitted in a Reptox
Binding site finder for best receptor ligang binding site.

\section{Binding residues}

A100 G104 D105 R106 E107 R108 G118 A119 G120 (Figure 5).

\section{Suitable Ligand Selection for Receptor Ligand Binding}

In this project the ligand selection for potential receptor based on ligand binding site and also available list favorable drugs for multiple myloma. Then select the potential ligand as drug candidate and change some confirmation on drug structure computationally then prepare final ligand for docking results analysis (Figure 6). 

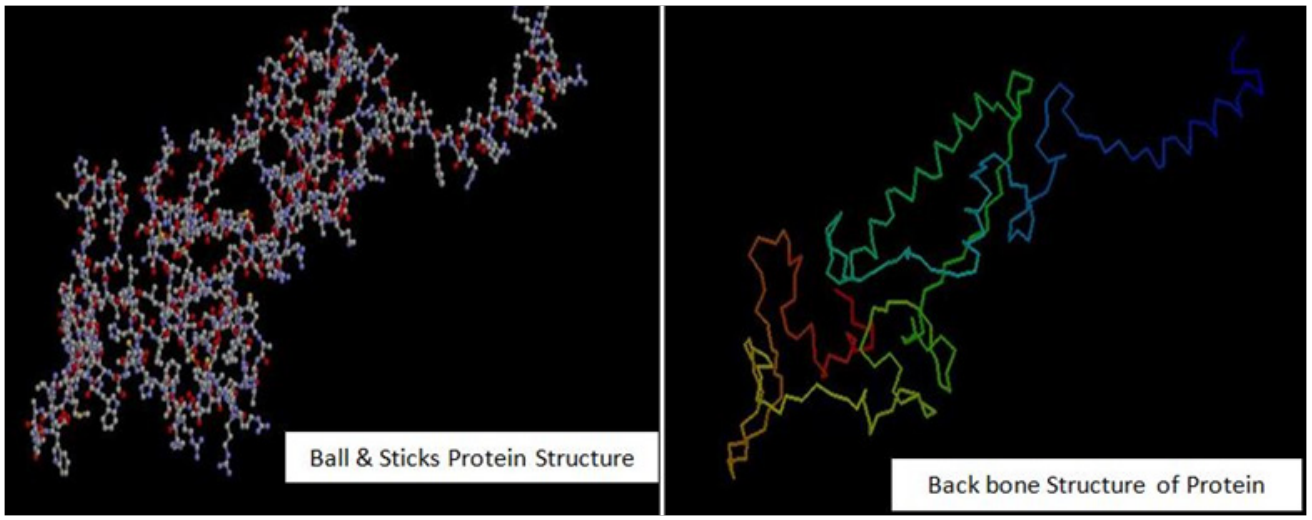

Figure 2 3D Structure of protein in a form of back bone \& ball sticks.
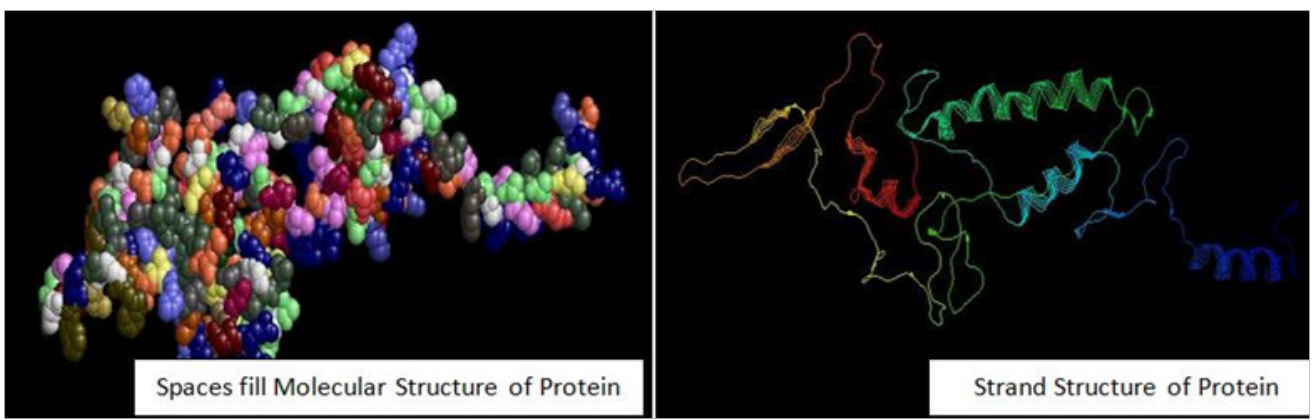

Figure 3 3D Structure of protein in a form of space fill \& strand structure.
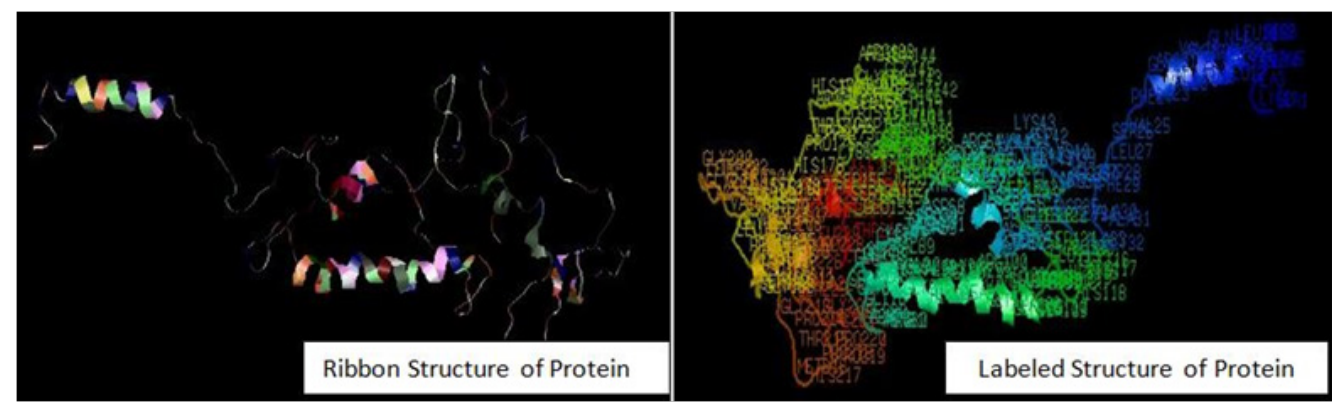

Figure 4 3D Structure of protein in a form of Ribbon \& labeled.

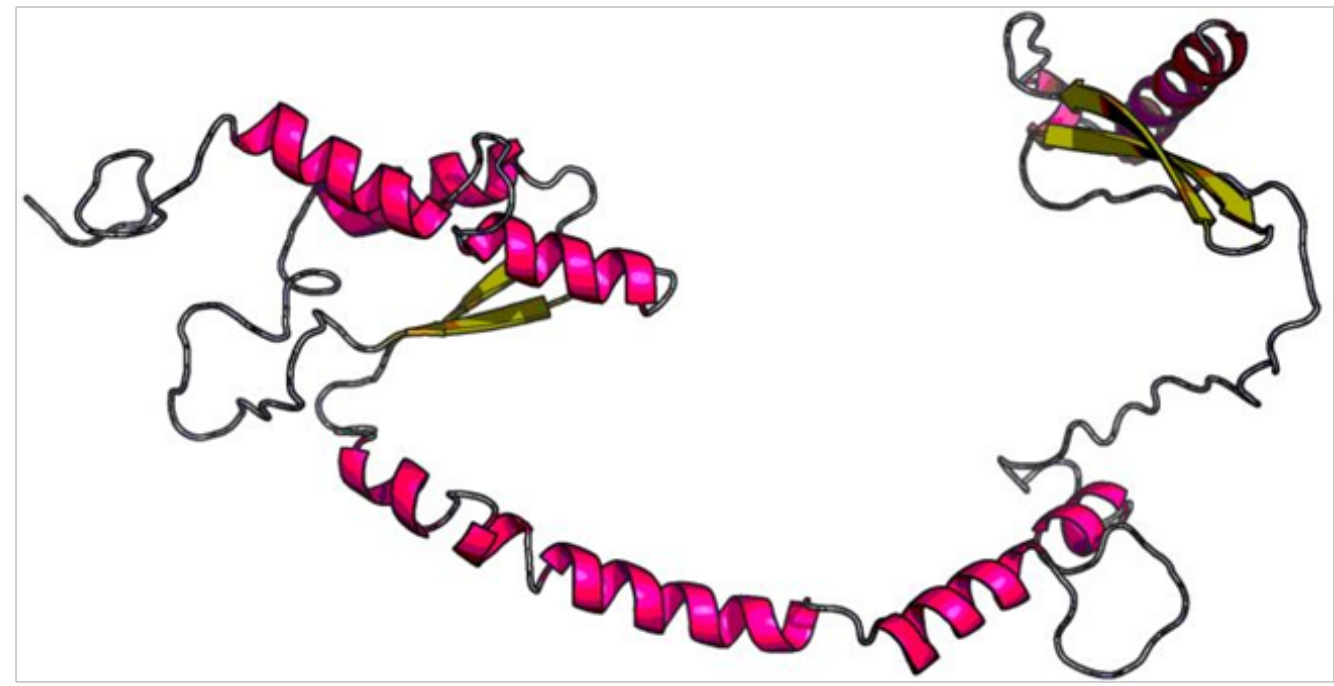

Figure 5 3D Structure of protein in a form of protein refined structure.

Citation: Srivastava V, Agrawal SP, Kalra SJS, et al. Computer aided drug designing for proteasome inhibitor to find out potential drug candidate for multiple myloma. MOJ Toxicol. 20I8;4(5):352-356. DOI: I0.15406/mojt.20I8.04.00I26 

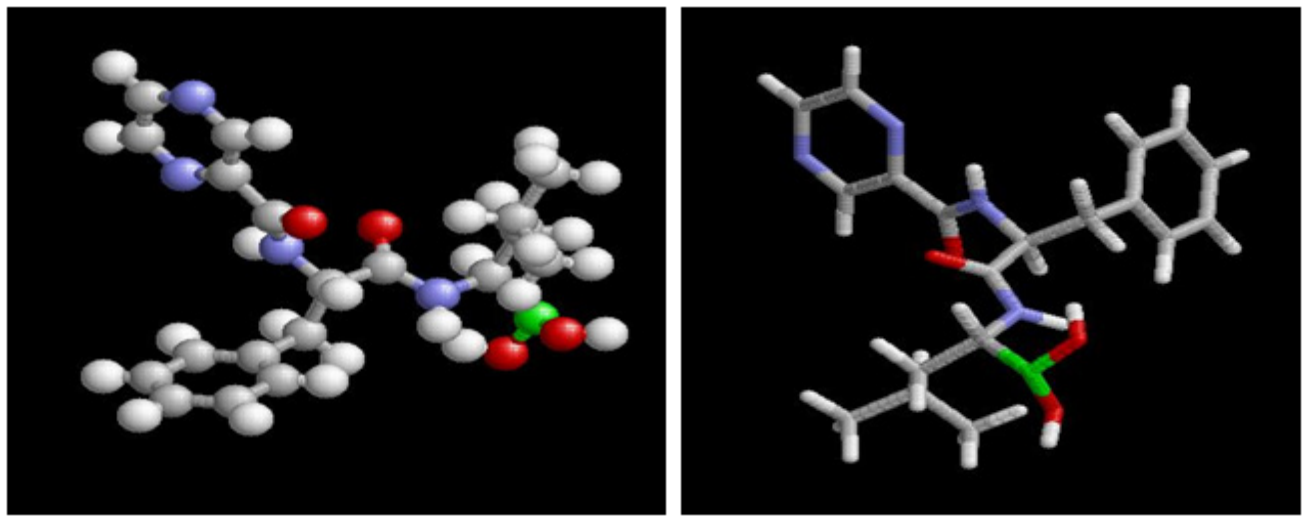

Figure 6 Structure of ligand with some conformational changes in main structure.

\section{Docking}

Prediction of the optimal physical configuration and energy between two molecules and the phenomenon which enables the interaction between receptor molecules and the ligand molecule, and mainly (Figure7) (Figure 8).
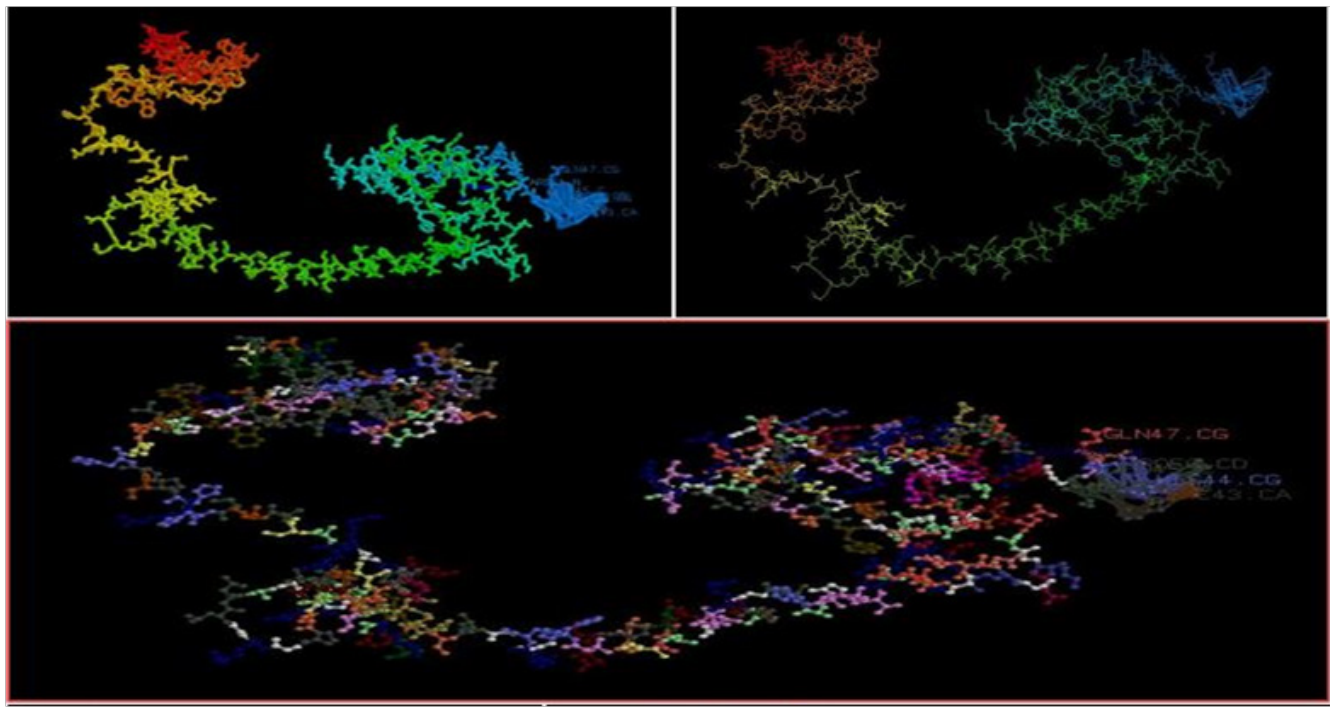

Figure 7 Different $3 \mathrm{D}$ model structure of docking model number I with ligand molecule.
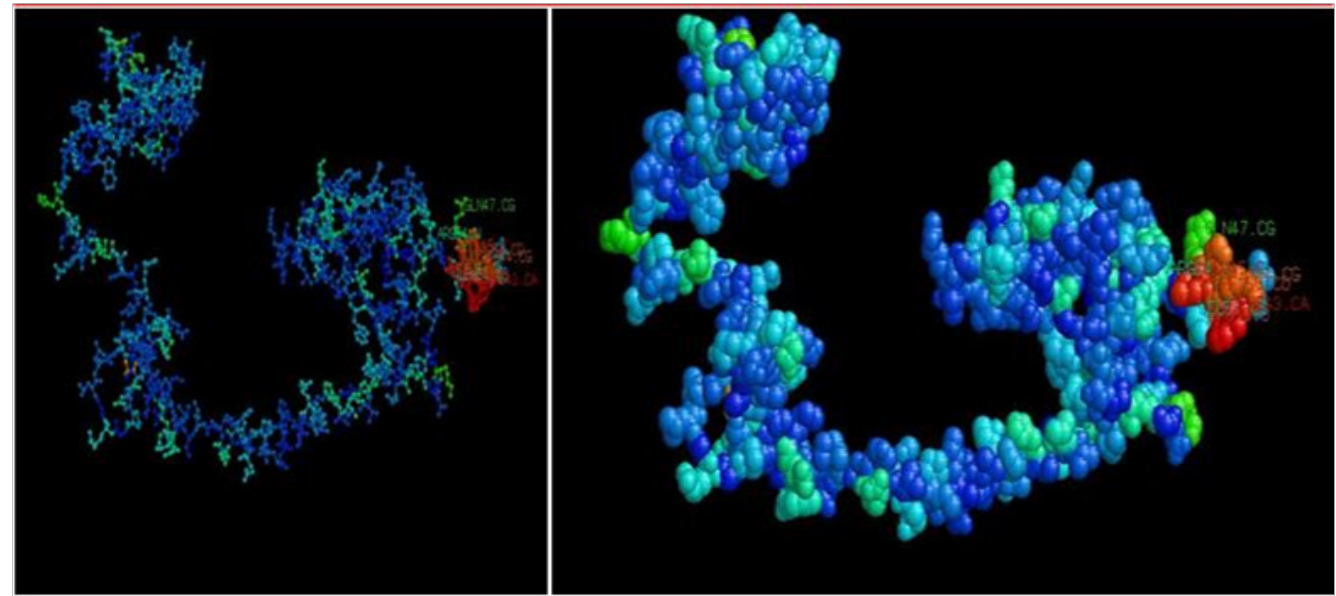

Figure 8 3D Structure of Docking model number I in a form of space fill \& ball sticks with ligand molecule.

\section{Conclusion}

Finally in this study of Proteasome protein structure that can be concluded10 number of model and 10 number of potential ligand with 3Dstructure which is predicted through the Insilico approaches and homology modeling and the docking of Proteasome protein with various ligand determine and the interaction between protein and ligand that's bind on active site of the Proteasome enzyme, although 
docking process. It is very complicated because its depends on various parameters the main resultant obtained by different type of docking tools and docking completed with the help of HEX,PATHADOCK for identify the suitable Proteasome inhibitors via different ligands which are docked with the Proteasome protein. Only 1 numbers of ligand given the minimum energy and out of these 10 ligands. Bortezomib is playing an important role in Multiple myloma and bortezomib ligand have minimum binding energy and its work as a potential Proteasome inhibitor as per the Table 1 parameters. Perhaps the ultimate solution is to develop a potential drug candidate against this devastating epidemic.

Table I Statistic calculation of 20 docking models

\begin{tabular}{|c|c|c|c|c|}
\hline Docking Model Number & Binding score & Area & ACE & Transformation information \\
\hline Docking Model I & 3682 & 468.70 & -192.32 & 0.94-0.44-3.0720.88- | 47.77| 45.00 (Clustering RMSD: 4.0) \\
\hline Docking Model 2 & 3636 & 430.80 & -178.17 & $-2.7|-0.8| \quad|.80| 43.35-66.4468 .0 \mid$ \\
\hline Docking Model 3 & 3490 & 384.30 & -144.34 & $0.43-0.85-0.3426 .2392 .4094 .25$ \\
\hline Docking Model 4 & 3480 & 437.50 & -183.08 & $2.21-0.35-0.59-50.43-95.75 \quad 176.70$ \\
\hline Docking Model 5 & 3380 & 423.60 & -128.38 & $-2.34 \mid .44-0.64-17.03-178.54-38.76$ \\
\hline Docking Model 6 & 3316 & 397.30 & -55.96 & $-0.290 .020 .70-118.589 .17-45.83$ \\
\hline Docking Model 7 & 3270 & 414.40 & -147.82 & $2.6|-0.20-2.42-4.5| \quad|3| .65 \mid 47.67$ \\
\hline Docking Model 8 & 3256 & 394.60 & -186.25 & $-0.13-0.76-0.6847 .04|83.0840 .8|$ \\
\hline Docking Model 9 & 3232 & 403.30 & -118.56 & 28.88 \\
\hline Docking Model 10 & 3218 & 407.60 & -91.55 & 3.010 .012 .65108 .4840 .2894 .74 \\
\hline Docking Model II & 3152 & 452.60 & -103.98 & $-0.64|.20-1.6572 .28-| 32.6 \mid-62.16$ \\
\hline Docking Model 12 & 3132 & 340.70 & -148.59 & $-1.98|.27-| .7|-97.76-| 20.8 \mid-57.48$ \\
\hline Docking Model I3 & 3100 & 394.40 & -135.21 & 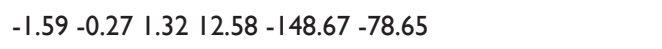 \\
\hline Docking Model 14 & 3086 & 437.40 & -97.04 & $-|.290 .2|-2.28|7.5|-27.40-|| 3.35$ \\
\hline Docking Model I5 & 3084 & 403.10 & -203.82 & $-1.660 .330 .7248 .43-16.98-105.44$ \\
\hline Docking Model I6 & 3070 & 380.50 & -172.17 & -0.220 .83 I. $14-103.6|23.33-5| .27$ \\
\hline Docking Model I7 & 3064 & 404.50 & -121.96 & $0.570 .31 \quad 1.12-164.56-66.8856 .60$ \\
\hline Docking Model 18 & 3056 & 417.90 & -81.88 & $0.90-1.022 .40-1|15.45-85.90| 44.86$ \\
\hline Docking Model 19 & 3016 & 380.60 & -121.62 & $-2.6|0.29| .39 \mid 23.67-107.94-12.27$ \\
\hline Docking Model 20 & 2996 & 373.30 & -49.59 & -3.09 I.05 I.34 I23.04 | |28.7|-10.05 \\
\hline
\end{tabular}

Table I Explanation : Table number I explained the statistic structure of bind ligand \& protein interaction total 20 structures found via this analysis but out of 20 only I number of docking model perfect as per the CADD parameters.

\section{Acknowledgements}

It is my proud privilege to express deepest sense of reverence and heart full thanks DBT, for giving me this platform I am extremely great full to Er. Neelesh Yadav from BIFC-FRI, Dehradun for giving me valuable guidance related to advance computer technology.

\section{Conflict interest}

None.

\section{References}

1. Teicher, Beverly A, Joseph E, et al. Proteasome inhibitors. Biochemical Pharmacology. 2015.

2. Pawlyn C, Morgan GJ. Evolutionary biology of high-risk multiple myeloma. Nat Rev Cancer. 2017;17(9):543-556.

3. Keiji Tanaka. Proteasomes: Structure and Biology. The Journal of Biochemistry. 1998;123(2):195-204.
4. Gelman JS, Sironi J, Berezniuk I, et al. Alterations of the intracellular peptidome in response to the proteasome inhibitor bortezomib. Plos One. 8(1):e53263.

5. www.ncbi.nlm.nihgov

6. www.pdb.org

7. www.drugbank.ca

8. blast.ncbi.nlm.nih.gov

9. nihserver.mbi.ucla.edu/SAVES/

10. www.modelling.leeds.ac.uk/qsitefinder/

11. Alka Dwivedi, Vijay Laxmi Saxena. In Silico Drug Designing of Protease Inhibitors to Find the Potential Drug Candidate for HIV1. Computational Biology and Bioinformatics. 2013;1(3):10-14.

12. http://www.polygenicpathways.co.uk/

13. Dubey A, Yadav N, Kalra SS. Analysis of protein modeling for envelope glycoprotein GP120 for HIV via bioinformatics approaches. International Research Journal of Virology. 2014;1(1):002-006. 